\title{
Post-biopsy renal arteriovenous fistula: successfully embolized with a Gianturco Mini Coil
}

\author{
M. J. D. CASSIDY \\ M.B., Ch.B., M.R.C.P. \\ E. P. HARRIES-JONES \\ M.B., B.Ch., F.R.C.P., A.B.D.R. \\ R. VAN ZYL-SMIT \\ M.B., B.Ch., F.C.P., M.R.C.P. \\ Renal Unit and Department of Radiology, Groote Schuur Hospital, Observatory, Cape Town, South Africa
}

\begin{abstract}
Summary
A case is reported of a renal arteriovenous fistula following percutaneous needle biopsy resulting in heavy haematuria that was successfully occluded by transcatheter embolization with a Gianturco Mini Coil.
\end{abstract}

\section{Introduction}

In recent years increasing numbers of percutaneous renal biopsies are being performed, occasionally resulting in complications such as arteriovenous (AV) fistulae. Over 35\% of all renal AV fistulae are formed secondary to percutaneous biopsy. In the past patients with bleeding fistulae were treated surgically, but more recently transcatheter embolization has become a recognized form of therapy. We describe below a case of post-biopsy renal arteriovenous fistula resulting in heavy haematuria and its successful occlusion by embolization with a Gianturco Mini Coil (Gianturco, Anderson \& Wallace, 1975).

\section{Case report}

A 22-year-old female was first noted to have microscopic haematuria following the delivery of her first child 17 months previously. Thirteen months later a percutaneous renal biopsy was done, following which she had heavy haematuria for 2 weeks, requiring blood transfusion. The renal biopsy showed a mild glomerulonephritis. Over the course of the next 3 months intermittent heavy macroscopic haematuria persisted, associated with the passage of clots.

She was admitted to Groote Schuur Hospital for investigation. She was generally well and had a blood pressure of $90 / 70 \mathrm{mmHg}$. Her urine contained numerous red cells, but no casts or protein. Her haemoglobin was $11.2 \mathrm{~g} / \mathrm{dl}$, serum urea $3.8 \mathrm{mmol} / 1$, ơ serum creatinine $99 \mu \mathrm{mol} / 1$. An excretory urogram was reported as normal.

A right renal arteriogram (Fig. 1) showed the presence of a large arteriovenous fistula centrally in $C_{D}$ the inferior half of the kidney. At the time of $\frac{O}{D}$ angiography it was decided to perform a therapeutic $\stackrel{\mathbb{D}}{3}$ transcatheter embolization of the AV fistula. A $5 \mathrm{~mm} \underset{\mathbb{D}}{\mathrm{C}}$ Gianturco Mini Coil was selectively placed into the enlarged artery feeding the AV fistula. A posi $\infty$ embolization arteriogram showed complete occlusion ? of the AV fistula with preservation of all the major peripheral branches of the right kidney (Fig. 2).

A few hours after the embolization, the patient experienced mild, transient loin pain and fever. $A \stackrel{2}{0}$ post-embolization renogram showed decreased per- $\varnothing$ fusion to the right lower pole, whilst a repeat $\underset{\vec{F}}{\overrightarrow{2}}$ excretory urogram failed to show any changes in the 옥 same region. No further macroscopic or microscopic haematuria has been noted during the follow up $\overrightarrow{0}$ period of ten months.

\section{Discussion}

The first cases of AV fistulae following percutaneous renal biopsy were described in 1962 (Boijsen and $₹$ Kohler, 1962; Fernstrom and Lindblom, 1962). Since 음 that time this abnormality has become recognized as $D$ a relatively common complication. Of 58 patients undergoing renal biopsy reported in 1965, 9 deve- $N$ loped AV fistulae (Bennet and Wiener, 1965). In man 0 over $35 \%$ of all renal fistulae are caused by biopsy. $\tilde{O}$ Experimentally AV fistulae may be produced in rabbits following needle biopsy. They are more likely $\sigma^{\circ}$ to occur in the medulla and the majority tend to close 0 spontaneously within two months (Ekelund, 1970).

Transcatheter embolization has been used with $\stackrel{?}{+}$ increasing frequency in an ever expanding list of 0 conditions in recent years. Besides its more tradi- 


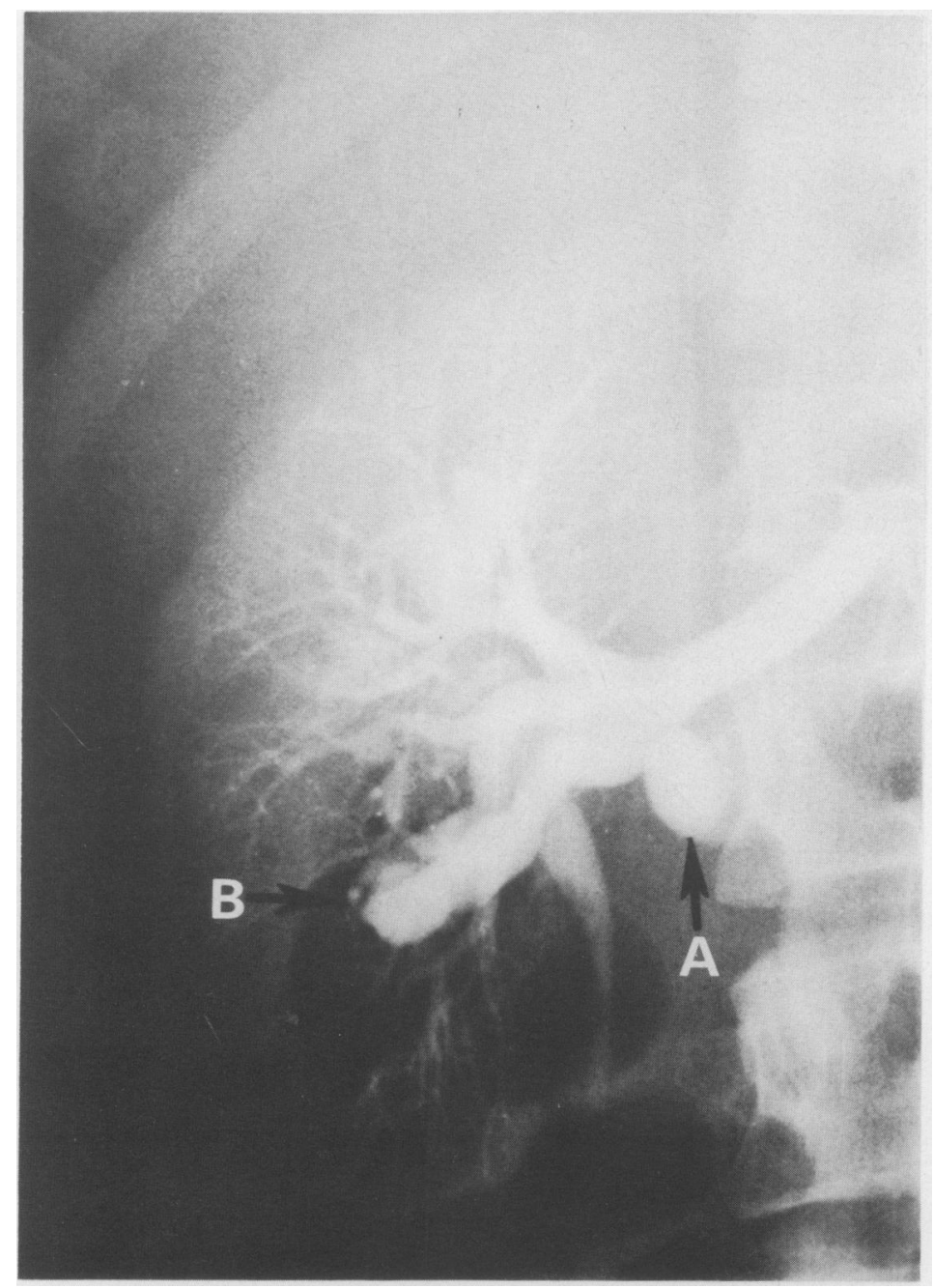

FIG. 1. Right renal arteriogram showing a large A-V fistula (B) with filling of the renal vein (A).

tional use in arteriovenous malformations and gastrointestinal arterial bleeds, great success has followed similar forms of treatment in traumatic vascular injury, metastatic liver disease, pulmonary arteriovenous malformations, primary tumours and in the treatment of intracranial aneurysms and cavernocarotid fistulae.

Angiographic embolic obliteration of renal arteriovenous malformations (AVM) has now become a recognized form of treatment of these lesions, and in most cases is an alternative to surgery. Autologous clot and other embolic materials including isobutyl2-cyanoacrylate (Gelfoam), detachable balloons and steel coils have been used (Greenfield, 1980; Pingoud, Glickman and Pias, 1980; White et al., 1980).
Whereas autologous clot and Gelfoam produce only temporary occlusion of arteries, the other embolic materials mentioned above cause permanent arterial occlusion. Permanent occlusive agents should be used in the treatment of AVMs. The beauty of the Gianturco Mini Coil is that it may be introduced through small diameter angiographic catheters, with a diameter as little as $5 \mathrm{~F}$, and can be very accurately placed within the orifice of the AVM. In performing this technique great care should be taken to ensure that the mini coil chosen has a slightly larger diameter than the feeding vessel in which it is to be placed. This precaution will prevent passage of the coil through the fistula, into the inferior vena cava and into the pulmonary circulation. 


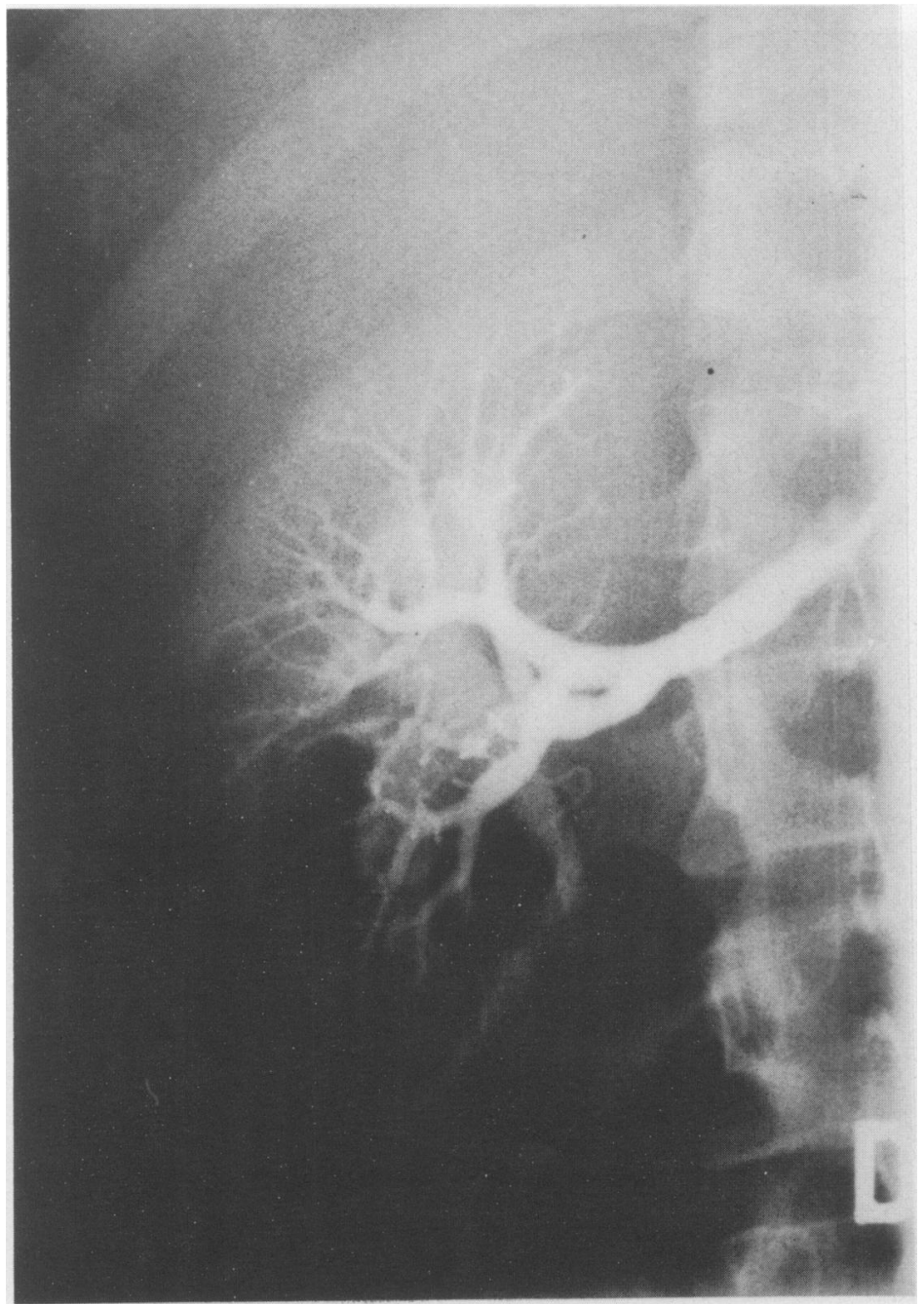

FIG. 2. Post-embolization arteriogram showing complete occlusion of the A-V fistula and preservation of the peripheral renal vasculature.

\section{References}

BENNET, A.R. \& WIENER, S.N. (1965) Intrarenal arteriovenous fistula and aneurysm. American Journal of Roentgenology, 95, 372.

BOIJSEN, E. \& KOHLER, R. (1962) Renal arteriovenous fistulae. Acta radiologica, 57, 433.

EKELUND, L. (1970) Arteriovenous fistulae secondary to renal biopsy. An experimental study in the rabbit. Acta radiologica (Diagnosis), 10, 218.

FERNSTROM, I. \& LINDBLOM, K. (1962) Selective renal biopsy using roentgen television control. Journal of Urology, 88, 709.
Gianturco, C., ANDerson, J.H. \& Wallace, S. (1975) Mechanical devices for arterial occlusion. American Journal of Roentgeno$\log y, 124,428$.

GREENFIELD, A.J. (1980) Transcatheter vessel occlusion: Selection 음 of materials. Cardiovascular and Interventional Radiology, 3, 222. Pingoud, E.G., Glickman, M.G. \& Pias, S.O. (1980) Ballooninduced thrombosis of renal arteriovenous fistula. American Journal of Roentgenology, 134, 605.

WhITE, R.J. JR, BARTH, K.H., KAUfFMAN, S.L., DECAPRIO, V. \& $\sigma$ SHANDBERG, J.D. (1980) Therapeutic embolisation with detach- N able balloons. Cardiovascular and Interventional. Radiology, 3, N 229. 